
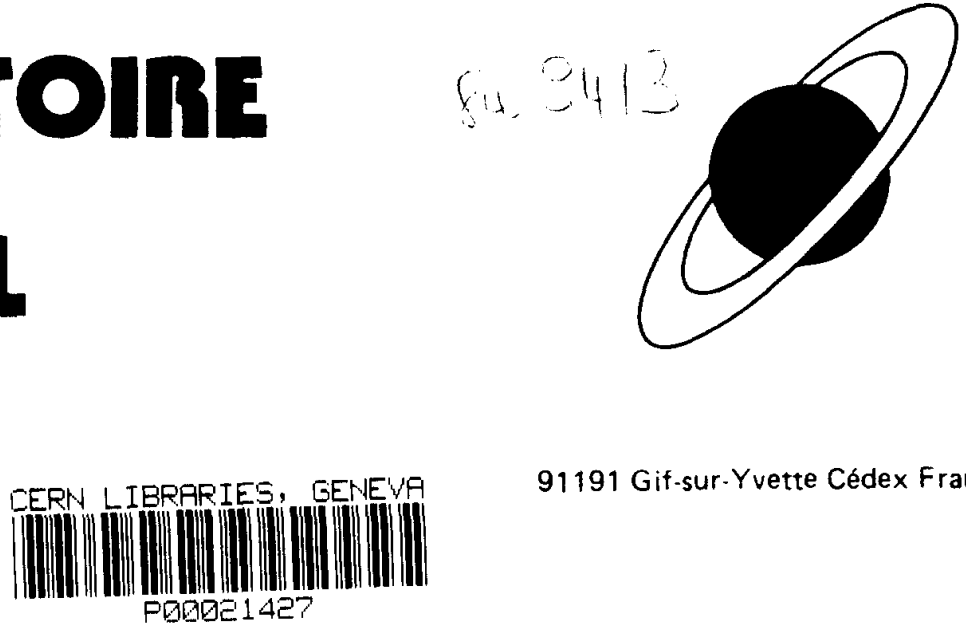

91191 Gif-sur-Yvette Cédex France

ANGULAR PATTERNS FROM FRAGMENTS PRODUCED IN CENTRAL HEAVY-ION COLLISIONS

S. R. Souza ${ }^{1}$, F. Schussler ${ }^{2}$, R. Donangelo ${ }^{3}$, K. Sneppen ${ }^{4}$, J. P. Bondorf ${ }^{4}$, R. Elmér ${ }^{5}$, B. Jakobsson ${ }^{5}$, S. Leray ${ }^{1}$, C. Ngô ${ }^{6}$, and H. Nifenecker ${ }^{2}$

${ }^{1}$ Laboratoire National Saturne, 91191 Gif Sur Yvette Cedex, France

${ }^{2}$ Institut des Sciences Nucléaires, 53 Avenue des Martyrs, Grenoble Cedex 38026, France

${ }^{3}$ Instituto de Física, Universidade Federal do Rio de Janeiro, 21945-970 Rio de Janeiro, Brazil

${ }^{4}$ The Niels Bohr Institute, Blegdamsvej 17, 2100 Copenhagen, Denmark

${ }^{5}$ University of Lund, Sölvegatan 14, 22362 Lund, Sweden

${ }^{6}$ Laboratoire d'Electronique, de Technologie et d'Instrumentation - DEIN/SPE 91191 Gif Sur Yevette Cedex, France

Submitted for publication to :

Physical Review C

L.NS/Ph/94-04

Centre National de la Recherche Scientifique cea 


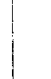




\title{
ANGULAR PATTERNS FROM FRAGMENTS PRODUCED IN CENTRAL HEAVY-ION COLLISIONS
}

S. R. Souza ${ }^{1}$, F. Schussler ${ }^{2}$, R. Donangelo ${ }^{3}$, K. Sneppen ${ }^{4}$, J. P. Bondorf ${ }^{4}$, R. Elmér ${ }^{5}$, B. Jakobsson ${ }^{5}$, S. Leray ${ }^{1}$, C. Ngô ${ }^{6}$, and H. Nifenecker ${ }^{2}$

${ }^{1}$ Laboratoire National Saturne, 91191 Gif Sur Yvette Cedex, France

2 Institut des Sciences Nucléaires, 53 Avenue des Martyrs, Grenoble Cedex 38026, France

${ }^{3}$ Instituto de Física, Universidade Federal do Rio de Janeiro, 21945-970 Rio de Janeiro, Brazil

${ }^{4}$ The Niels Bohr Institute, Blegdamsvej 17, 2100 Copenhagen, Denmark

${ }^{5}$ University of Lund, Sölvegatan 14, 22362 Lund, Sweden

${ }^{6}$ Laboratoire d'Electronique, de Technologie et d'Instrumentation - DEIN/SPE

91191 Gif Sur Yevette Cedex, France

\begin{abstract}
The angular patterns of fragments produced in central heavy-ion collisions at intermediate bombarding energies, and observed in emulsion experiments, are analyzed through simulations based on a molecular dynamical approach. The results indicate that these angular patterns give information on the shape of the multifragmenting system at relatively early stages of the collision process.
\end{abstract}

The break-up of nuclear systems into several fragments of intermediate sizes (IMF) is the most important reaction mechanism in central collisions at intermediate bombarding energies. (See [1-7] and references therein). Despite large efforts in collecting information concerning the multifragmentation process, the mechanisms that cause nuclei to break-up into several pieces have not yet been completely elucidated. (See [8-10] 
for recent reviews on the subject). In this context, emulsion experiments play an important role because of the full detail with which multifragmentation events can be reconstructed [1]. Indeed, their very low detection thresholds and full $4 \pi$ angular coverage make it possible to identify the atomic numbers $Z$ of all fragments (and for $Z=1,2$ also their masses), as well as to determine their momenta. In the present work we show how these data provide information on the shape of nuclear systems during the stages of the reaction at which fragments are produced.

The possibility, based on theoretical grounds, that exotic density shapes (bubbles, rings, and disks) appear after the most violent stages of the collision brought much attention lately [11-17]. Even more recently, Batko and Randrup [18] suggested that the angular distribution of fragments could give information on the shape of the system in early stages of the reaction. This pattern could thus help ascertain the appearance of such exotic density shapes, providing in that way further information about the nuclear equation of state $[15,17]$.

The shape of the nuclear system can be characterized by the multipolarity coefficients [18],

$$
a_{L M}=\frac{1}{A} \int d^{3} \mathbf{r} \rho(\mathbf{r}) Y_{L M}(\hat{\mathbf{r}}),
$$

where $A$ stands for the mass number of the total system, $\rho(\mathbf{r})$ the nuclear density and $Y_{L M}(\hat{\mathbf{r}})$ the usual spherical harmonics. The multipolarity parameters $a_{L M}$ are complex quantities. As suggested in ref. [18], it is more natural to work with the real parameters,

$$
b_{L M}=\frac{1}{2}\left(\left|a_{L M}\right|^{2}+\left|a_{L-M}\right|^{2}\right) \text {. }
$$

The $b_{L M}$ coefficients can be obtained readily from any dynamical approach, since their determination only requires knowledge of the nuclear density, which is on hand in all such models. However, when confronting data to the predictions of different models, one should carefully consider the definition of the angles entering in equation (1). In order to avoid ambiguities, we defined the polar and azimuthal angles in the frame in which the $x, y, z$ axes are parallel to the eigenvectors of the momentum tensor, and ordered according to increasing eigenvalues. 
At this point, one should remember that experiments provide us only with information about the charge (or mass) of the fragments and their asymptotic momenta. Assuming that the angular distribution of neutrons is similar to that of the protons, the multipolarity coefficients for the fragment angular distribution can be defined in an analogous manner,

$$
\alpha_{L M}=\frac{1}{Z} \sum_{i=1}^{N_{C}} Z_{i} Y_{L M}\left(\hat{\mathbf{p}}_{i}\right)
$$

and

$$
\lambda_{L M}=\frac{1}{2}\left(\left|\alpha_{L M}\right|^{2}+\left|\alpha_{L-M}\right|^{2}\right)
$$

where $N_{C}$ stands for the charge multiplicity, $Z_{i}$ the atomic number of the $i$ th fragment, $\hat{\mathbf{p}}_{\boldsymbol{i}}$ its unit momentum vector, and $Z$ the atomic number of the total system.

We must now study the connection between the multipolarity coefficients $\lambda_{L M}$ and $b_{L M}$, and, in particular, whether they contain the same information. In order to investigate this point we have used a molecular dynamical (M.D.) approach $[19,20]$ which allows one to address, at least qualitatively, the fragment formation process. In this treatment the time evolution of the $A$ nucleons is governed by Hamilton equations of motion. Hard collisions between particles are allowed, depending upon the phase space occupation in the neighborhood of the colliding nucleons. Since this approach allows one to follow the dynamical evolution of the system from the early stages of the reaction to configurations in which the fragments are far apart from each other, we have calculated the multipolarity coefficients for the three following cases:

(i) in position space at $200 \mathrm{fm} / \mathrm{c}$,

(ii) in momentum space at $200 \mathrm{fm} / \mathrm{c}$, and

(iii) in momentum space at an infinite time.

In the calculations in (ii) and (iii) eqs. (3) and (4) are used, whereas the multipolarity coefficients in (i) are obtained through $a_{L M}=1 / Z \sum_{i} Z_{i} Y_{L M}\left(\hat{\mathbf{r}}_{i}\right)$, where $\hat{\mathbf{r}}_{i}$ is the unit position vector of the $i$ th cluster.

The results of the numerical simulations are shown in Figs. 1a, 1b, and 1c, for the three different systems analyzed in the emulsion experiment: $\mathrm{Kr}$ (95 $\mathrm{MeV}$ per 
nucleon $)+\operatorname{AgBr}[7,21], \operatorname{Ar}(65 \mathrm{MeV}$ per nucleon $)+\operatorname{AgBr}[6,21,22]$, and $\mathrm{O}(210 \mathrm{MeV}$ per nucleon $)+\operatorname{AgBr}[1,6,22,23]$. The full symbols in this picture represend the results obtained according to (i), while the open circles and squares correspond to (ii) and (iii), respectively. In spite of some discrepancies, these calculations seem to indicate that information about density shapes can be equally well obtained from analyses in either momentum or position space. Furthermore, the good overall agreement between the multipolarity coefficients obtained at $200 \mathrm{fm} / \mathrm{c}$ with those calculated at an infinite time indicates that the latter retains information on what happened during relatively early stages of the reaction. Thus, one can conclude from the results presented here that information about density shapes appears not to be washed out due to propagation in the Coulomb field. We could not investigate whether this conclusion still holds much earlier than $200 \mathrm{fm} / \mathrm{c}$ because of the difficulty in identifying fragments in a reliable way at the very early stages of the collision, when they are not yet sufficiently separated in space.

Before going any further, it is useful to give some information concerning the geometrical cross-section associated with the central events we are going to discuss. Owing to the low statistics of emulsion data, it is difficult to obtain very precise impact parameter ranges from the available methods usually employed in the selection of central collisions. In ref. [1], the multiplicity of charged particles was used to sort out the most central collisions. By assuming a smooth functional dependence of the charge multiplicity on the impact parameter, it is possible to estimate the fraction of the total cross-section which is associated with these central events. (See [5] and references therein). From analyses based on this method, the maximum impact parameter $b_{\max }$ associated with the central events we are going to discuss is $b_{\max } \approx 1.5 \mathrm{fm}$. This is a reliable method for high energy heavy-ion collisions [24], but it is not very well suited for reactions at lower energies $[6,24]$, such as $\operatorname{Ar}(65 \mathrm{MeV}$ per nucleon) and $\operatorname{Kr}(95 \mathrm{MeV}$ per nucleon) $+\mathrm{AgBr}$, we are going to consider below. Thus one should bear in mind the fact that $b_{\max } \approx 1.5 \mathrm{fm}$ gives only an estimate of the maximum impact parameter associated with the experimental events we are going to discuss. We shall come back to 
this point latter on.

We have shown that the multipolarity coefficients $\lambda_{L M}$ provide information on the shape of the event at the moment the system breaks up and the fragments start to move away from each other under the influence of the Coulomb repulsion. Let us now compare the results of our dynamical simulations with experimental emulsion data, together with a Monte Carlo (M.C.) calculation in which the emission angles of the fragments (observed experimentally) are chosen at random. The results for very central collisions (see ref. [22] and discussion below) between $\mathrm{Kr}+\mathrm{AgBr}$ at $95 \mathrm{MeV}$ per nucleon are shown in Fig. 2. We see that, in all cases, the multipolarity coefficients $\lambda_{L M}$ are independent of $M$. The fact that the experimental distribution agrees with the Monte Carlo simulation indicates that the fragments are emitted isotropically. We have checked that this is supported by the model simulation which gives a fairly isotropic distribution of fragments. This would not be the case if the break up of the system occurred in a configuration such that the nuclear density is fairly oblate. Intuitively, and already discussed in ref. [15], all the fragments originated from the break up of doughnut or disk shaped densities should be observed nearly in the same plane. That would certainly lead to a strong functional dependence of $\lambda_{L M}$ on $M$, in contrast with the behavior shown in Fig. 2. Thus, it turns out from this analysis that the nuclear density is fairly spherical at the moment the fragments are formed in the most central collisions between $\mathrm{Kr}(95 \mathrm{MeV}$ per nucleon $)+\mathrm{AgBr}$ nuclei.

Figure 3 shows the multipolarity coefficients for central collisions between the $\mathrm{Ar}+\mathrm{AgBr}$ system at $65 \mathrm{MeV}$ per nucleon. The results indicate that the situation is different from the previous case. Here the predictions of the molecular dynamical calculation agree quite well with the Monte Carlo simulation, but both disagree with the data. The dependence of the experimental multipolarity coefficients $\lambda_{L M}$ on $M$ seems to indicate that the events are slightly disk shaped. This could be interpreted as a possible signature of doughnut or disk shaped densities at the later stages of the nuclear multifragmentation process. However, there is a more likely explanation to the angular pattern suggested by the multipolarity coefficients shown in Fig. 3. This feature 
can be understood by considering the criterion employed in the selection of the most central events used in this analysis. They have been chosen by imposing the following conditions $[6,22]$ :

(i) No heavy remnant $(Z \leq 15)$, and

(ii) Elongation parameter $\beta<0.25$. This parameter is defined as $\beta=\left(\lambda_{1}-\lambda_{2}\right) /\left(\lambda_{1}+\right.$ $\lambda_{2}+\lambda_{3}$ ), where $\lambda_{1}>\lambda_{2}>\lambda_{3}$ are the eigenvalues of the momentum tensor.

As is discussed in ref. [22], this is a strong centrality criterion, much stronger than those based on the charge multiplicity. Nevertheless, since there is no cross-section associated with zero impact parameter, the collisions must take place at some finite value of the impact parameter. Furthermore, the growth of dynamical fluctuations could mix up collisions that occur at slightly different impact parameters. In order to check whether the increase of $\lambda_{L M}$ as a function of $M$ can be accounted for by these hypotheses we have performed numerical simulations using the molecular dynamical approach.

The results are presented in Fig. 4, where the elongation parameter $\beta$ is calculated for collisions taking place at impact parameters equal to 0,1 and $2 \mathrm{fm}$. The shaded area represents the events that would be accepted according to the centrality criterion (ii). One sees that this number is $90 \%$ at $b=0 \mathrm{fm}, 78 \%$ at $b=1 \mathrm{fm}$ and $63 \%$ at $b=2 \mathrm{fm}$. Thus, the behavior of the multipolarity coefficients $\lambda_{L M}$ shown in Fig. 3, might be due to finite values of the impact parameters. In fact, Fig. 5 shows a comparison between the experimental data and the results of molecular dynamics for collisions taking place at $b=1$ and $2 \mathrm{fm}$. In this case there is a very good overall agreement with experiment. This confirms the hypothesis that the slight increase of $\lambda_{L M}$ as a function of $M$ is only due to the fact that collisions take place at non-zero impact parameters. Disk shaped events have been predicted for this system at the bombarding energy considered here in recent investigations based on the Boltzmann-Uehling-Uhlenbeck (BUU) approach if a stiff equation of state is assumed [17]. On the other hand, it has been found that a soft equation of state would lead to a radial expansion through bubble formation [17]. Even though one cannot draw conclusions about the growth of bubbles from the analysis presented here, in spite of being consistent with the large radial expansion observed in 
this reaction and already reported in ref. [6], the hypothesis of disk shaped densities seems to be eliminated. Thus, our results seem to favor a soft equation of state for nuclear matter.

We consider, finally, very central collisions between $\mathrm{O}+\mathrm{AgBr}$ at $210 \mathrm{MeV}$ per nucleon. The results are shown in Fig. 6. For this system the Monte Carlo simulation agrees quite well with data, suggesting an isotropic emission of fragments, while the predictions of molecular dynamics disagree completely. Figure 7 illustrates the molecular dynamical system at $200 \mathrm{fm} / \mathrm{c}$ in the center-of-mass reference frame, taking the $\mathbf{x}$-axis parallel to the beam. The nucleon distribution is fairly isotropic in the plane perpendicular to the beam axis (the $y-z$ plane). However, there is a forward elongation of the density along the beam axis. This leads to the calculated multipolarity coefficients $\lambda_{L M}$ in Fig. 6, which correspond to a forward emission of fragments. The fact that this result stands in clear conflict with experimental observations, as shown in Fig. 6, could lead one to the conclusion that the initial translational kinetic energy is not correctly converted into excitation in the molecular dynamical simulation. More precisely, if a larger amount of the kinetic energy associated with the longitudinal motion were transferred to the transverse motion, there would be less fragments emitted in the forward direction. Thus the shape of the system would look more spherical and would agree better with the experimental results.

This forward emission of fragments is not surprising at this bombarding energy since, because of the violence of the collision, the system should not have time to attain a large degree of equilibration before the disassembly process takes place. Therefore, some nucleons and fragments should keep a certain memory from very early stages of the reaction. Nevertheless, in view of the disagreements between the experimental and calculated multipolarity coefficients, it seems that some other point is missing in the analysis. As in the reaction considered previously, effects associated with the non-zero values of the impact parameters might be able to harmonize this picture with the experimental observations shown in Fig. 6. Indeed, for actual nucleus-nucleus collisions there is always a privileged plane in the position space, the reaction plane, defined by 
the relative spatial orientation of the colliding nuclei at the beginning of the reaction. We have seen from the results presented in Fig. 5 that this leads to a correlation between the fragments in this plane. Thus, this effect could change the behavior of the multipolarity coefficients predicted by the molecular dynamical approach shown in Fig. 6 . This is illustrated in Fig. 8, which shows a comparison between the experimental data and the model simulations for collisions at impact parameters $b=1 \mathrm{fm}$ and $b=2 \mathrm{fm}$. Theses results show that the agreement with the experimental data is clearly improved. Moreover, the systematic deviations of the calculated multipolarity coefficients, for collisions at $b=2 \mathrm{fm}$, from the experimental ones seem to indicate that these collisions occur within the window of impact parameters $0 \leq b \leq 1 \mathrm{fm}$. We have also checked that the centrality criterion (ii), illustrated in Fig. 4 for the $\mathrm{Ar}+\mathrm{Ag}$ system, is fulfilled by $80 \%$ and $60 \%$ of the events for impact parameters equal to 1 and $2 \mathrm{fm}$, respectively. Thus, the molecular dynamical simulations suggest that effects associated with nonzero impact parameters can prevent aspects related to the forward emission shown in Fig. 6 from manifesting in the multipolarity coefficients. It is useful to mention that estimates based on the multiplicity of charged particles, which should be a good impact parameter filter at this bombarding energy [24], give $b_{\max } \approx 0.8 \mathrm{fm}$.

In conclusion, we have checked by means of dynamical simulations that the analysis of angular patterns gives valuable information on the shape of the nuclear density at relatively early stages of the reaction. Comparison of the predictions of the model simulation with experimental data shows that care should be taken in associating these angular patterns with a particular density shape, because the slight non-centrality of the collisions can conceal the actual shape of the event. The fact that the multipolarity coefficients are quite sensitive to such fine aspects, such as small impact parameters, shows that this kind of analysis is very well suited for emulsion experiments. In fact, because of the full $4 \pi$ angular coverage, emulsion data are not biased by geometrical limitations that would certainly distort the behavior of the multipolarity coefficients. Furthermore, since the shape of the density at the moment at which the system disassembles should be strongly dependent on the equation of state for nuclear matter, as 
well as on nucleon-nucleon collisions, this kind of analysis could help understand these quantities through studies based on dynamical approaches. In this context, the results obtained here for collisions between $\mathrm{Kr}+\mathrm{AgBr}$ and $\mathrm{Ar}+\mathrm{AgBr}$ nuclei, at bombarding energies below $100 \mathrm{MeV}$ per nucleon, reveal that fragments should be isotropically emitted in perfectly central reactions. This gives indications that disk (or ring) shaped events do not happen in these reactions and therefore a stiff equation of state for nuclear matter does not seem to be supported by the analysis, in accordance to the theoretical considerations made in $[15,17]$.

K. Sneppen thanks financial support from the Carlsberg Foundation. R. Donangelo and S. R. Souza acknowledge finantial support from $\mathrm{CNPq}$ - Conselho Nacional de Desenvolvimento Científico e Tecnológico - Brazil, as well as from the Niels Bohr Institute, and thank it for the warm hospitality extended to them. We thank the Danish Natural Science Research Council for support. 


\section{References}

[1] B. Jakobsson et al., Nucl. Phys. A509, 195 (1990).

[2] M. T. Tsang et al., Phys. Rev. Lett. 71, 1502 (1993).

[3] R. T. de Souza et al., Phys. Lett. B 268, 6 (1991)

[4] P. Kreutz et al., Nucl. Phys. A556, 672 (1993).

[5] J. Hubele et al., Z. Phys. A 340, 263 (1991).

[6] H. W. Barz et al., Nucl. Phys. A531, 453 (1991).

[7] W. Bauer, J. P. Bondorf, R. Donangelo, R. Elmer, B. Jakobsson, H. Schulz, F. Schussler, and K. Sneppen, Phys. Rev. C 47, R1838 (1993).

[8] L. G. Moretto and G. J. Wozniak, Ann. Rev. Nucl. Part. Sci. 43, 379 (1993).

[9] J. Aichelin, Phys. Rep. 202, 233 (1991).

[10] D. H. E. Gross, Rep. Prog. Phys. 53, 605 (1990).

[11] L. Moretto, Kin Tso, N. Colonna, and G. J. Wozniak, Phys. Rev. Lett. 691884 (1992).

[12] W. Bauer, G. F. Bertsch, and H. Schulz, Phys. Rev. Lett. 69, 1888 (1992).

[13] C. E. Aguiar, R. Donangelo, C. O. Dorso, R. S. Gomes, and N. Gonçalves, Phys. Rev. C 46, 1069 (1992).

[14] B. Borderie, B. Remaud, M. F. Rivet, and F. Sebille, Phys. Lett. B 302, 15 (1993).

[15] H. M. Xu, J. B. Natowitz, C. A. Gagliardi, R. E. Tribble, C. Y. Wong, and W. G. Lynch, Phys. Rev. C 48, 933 (1993).

[16] H. Ngô, F. Z. Ighezou, C. Ngô, J. Nemeth, and L. De Paula, in Proceedings of the XXI Workshop on Gross Properties of Nuclei and Nuclear Excitations, Hirschegg, 1993, editted by H. Feldmeier (GSI, Darmstadt, 1993) p. 302.

[17] S. R. Souza and C. Ngô, Phys. Rev. C 48, R2555 (1993).

[18] G. Batko and J. Randrup, Nucl. Phys. A563, 97 (1993).

[19] S. R. Souza, L. De Paula, S. Leray, J. Nemeth, C. Ngô, and H. Ngô, in proceedings of the XXX International Meeting on Nuclear Physics, Bormio, 1992, editted by I. Iori (University of Milano, Milano, 1992) p. 45. 
[20] S. R. Souza, L. De Paula, S. Leray, J. Nemeth, C. Ngô, and H. Ngô, Nucl. Phys. A, (in press) (1994).

[21] F. Schussler et al., (unpublished).

[22] H. W. Barz et al., Nucl. Phys. A548, 427 (1992)

[23] F. Schussler, E. Monnand, H. Nifenecker, B. Jakobsson, G. Jönsson, L. Karlsson, V. Kopljar, B. Norén, and K. Söderström, Report (Lund) LUIP-8805, 1988.

[24] L. Zhuzia, C. Hartnack, H. Stöcker, and W. Greiner, Phys. Rev. C 44, 824 (1991). 


\section{FIGURE CAPTIONS}

Fig. 1a) Multipolarity coefficients obtained from molecular dynamical simulations of head-on collisions between $\mathrm{Kr}+\mathrm{AgBr}$ at $95 \mathrm{MeV}$ per nucleon. The full circles stand for the results of the model simulation when the multipolarity coefficients are calculated using the spatial distribution of fragments at $200 \mathrm{fm} / \mathrm{c}$. Multipolarity coefficients obtained from the analysis in momentum space at $200 \mathrm{fm} / \mathrm{c}$ and at an infinite time are represented by open circles and squares, respectively.

Fig. 1b) Same as Fig. 1a, but the system is $\mathrm{Ar}(65 \mathrm{MeV}$ per nucleon $)+\mathrm{AgBr}$.

Fig. 1c) Same as Fig. 1a, but the system is $\mathrm{O}(210 \mathrm{MeV}$ per nucleon $)+\mathrm{AgBr}$.

Fig. 2) Multipolarity coefficients from the reaction $\mathrm{Kr}(95 \mathrm{MeV}$ per nucleon) $+\mathrm{AgBr}$. The full circles denote the experimental coefficients obtained from emulsion data, whereas the open circles stand for a Monte Carlo simulation (see text). The predictions of the molecular dynamical model, for head - on collisions, are represented by the open squares.

Fig. 3) Same as Fig. 2, but the system is $\mathrm{Ar}(65 \mathrm{MeV}$ per nucleon) $+\mathrm{AgBr}$.

Fig. 4) Elongation parameter $\beta$ obtained from simulations based on the molecular dynamical approach for collisions between $\mathrm{Ar}$ (65 $\mathrm{MeV}$ per nucleon) $+\mathrm{Ag}$ at impact parameters equal to 0,1 and $2 \mathrm{fm}$.

Fig. 5) Experimental multipolarity coefficients (full circles) from reactions between $\mathrm{Ar}$ (65 $\mathrm{MeV}$ per nucleon) $+\mathrm{AgBr}$ compared with collisions simulated by the molecular dynamical approach at impact parameters equal to 1 and $2 \mathrm{fm}$, open circles and squares, respectively.

Fig. 6) Same as Fig. 2, but the system is $\mathrm{O}(210 \mathrm{MeV}$ per nucleon $)+\mathrm{AgBr}$. 
Fig. 7) Illustration of the nucleon distribution in position space at $200 \mathrm{fm} / \mathrm{c}$ in a head-on collision between $\mathrm{O}+\mathrm{Ag}$ nuclei using molecular dynamics. The $y-z$ plane is perpendicular to the beam axis. The projectile hits the target coming from $x=-\infty$ to $+\infty$. Full circles correspond to nucleons that initially belonged to the ${ }^{16} \mathrm{O}$ nucleus, while open circles are associated with the Ag nucleus.

Fig. 8) Same as Fig. 5, but the system is $\mathrm{O}(210 \mathrm{MeV}$ per nucleon $)+\mathrm{AgBr}$. 


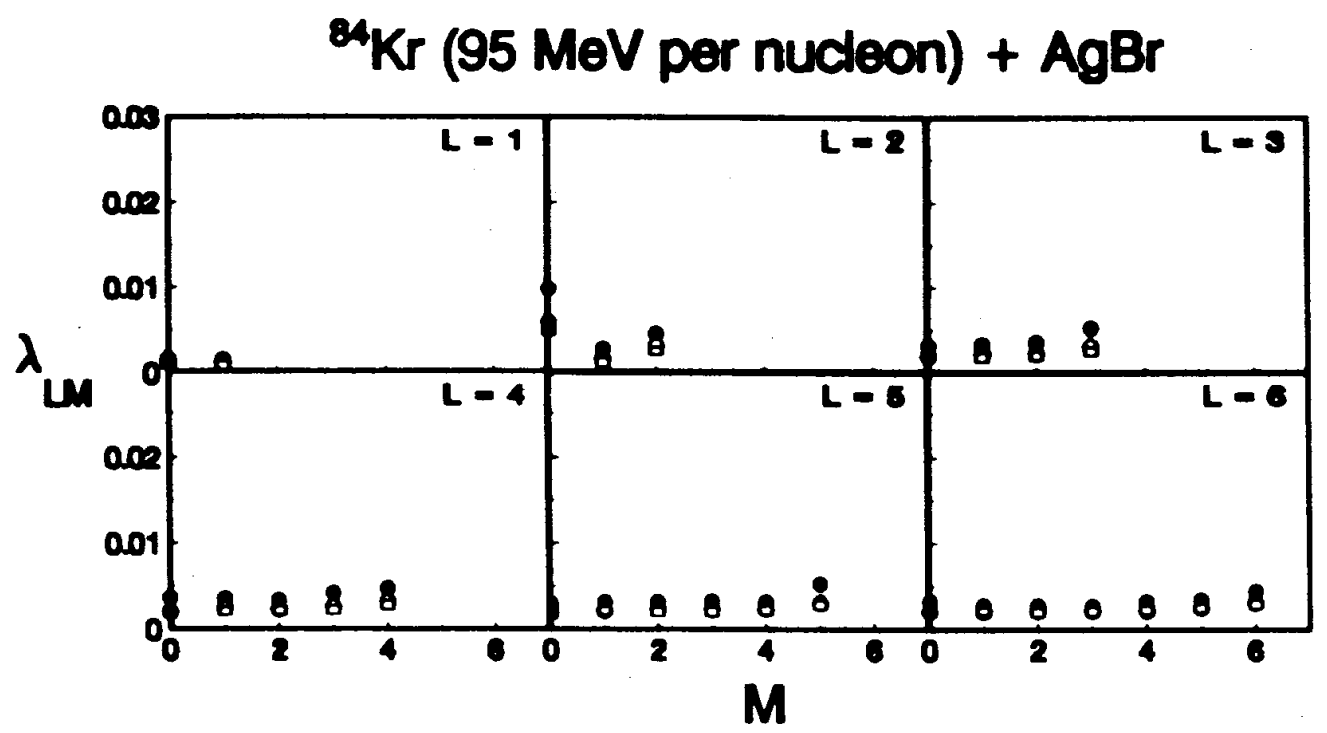

Fig. 10 


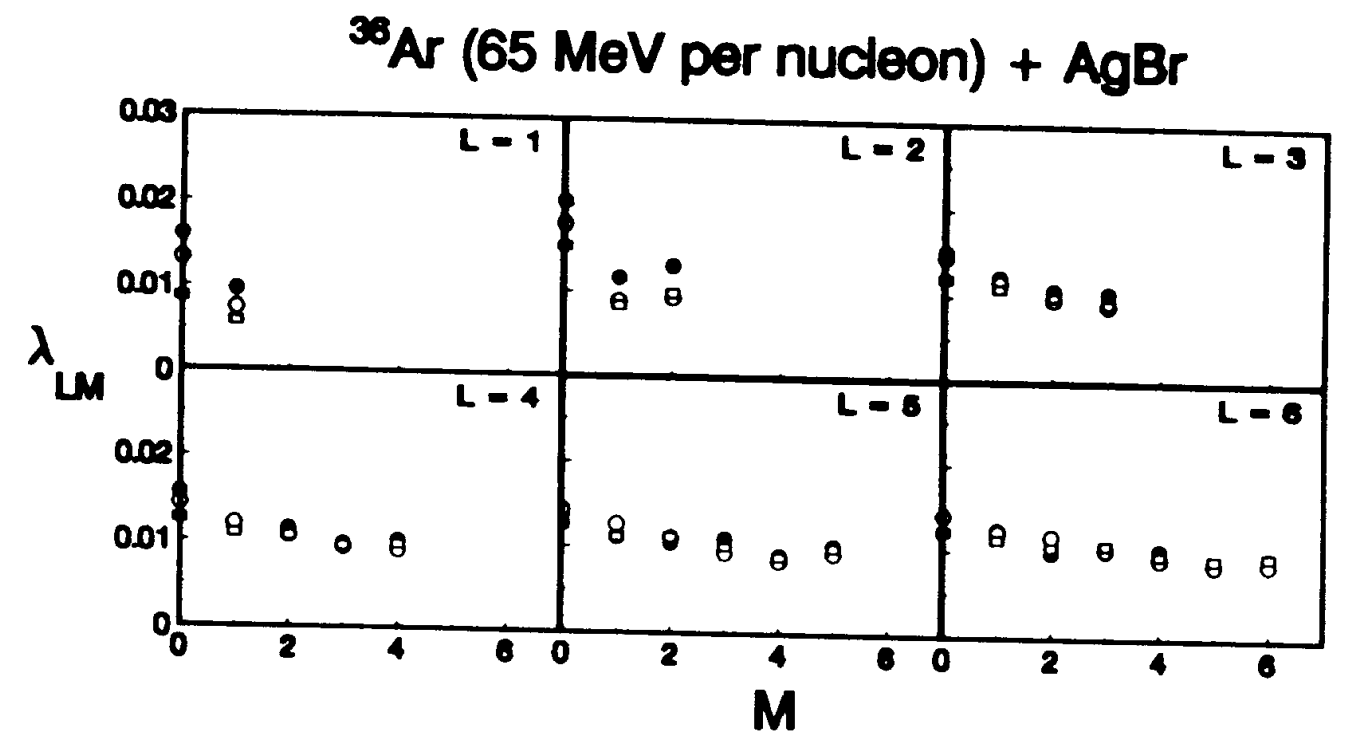

Fig. Ib 


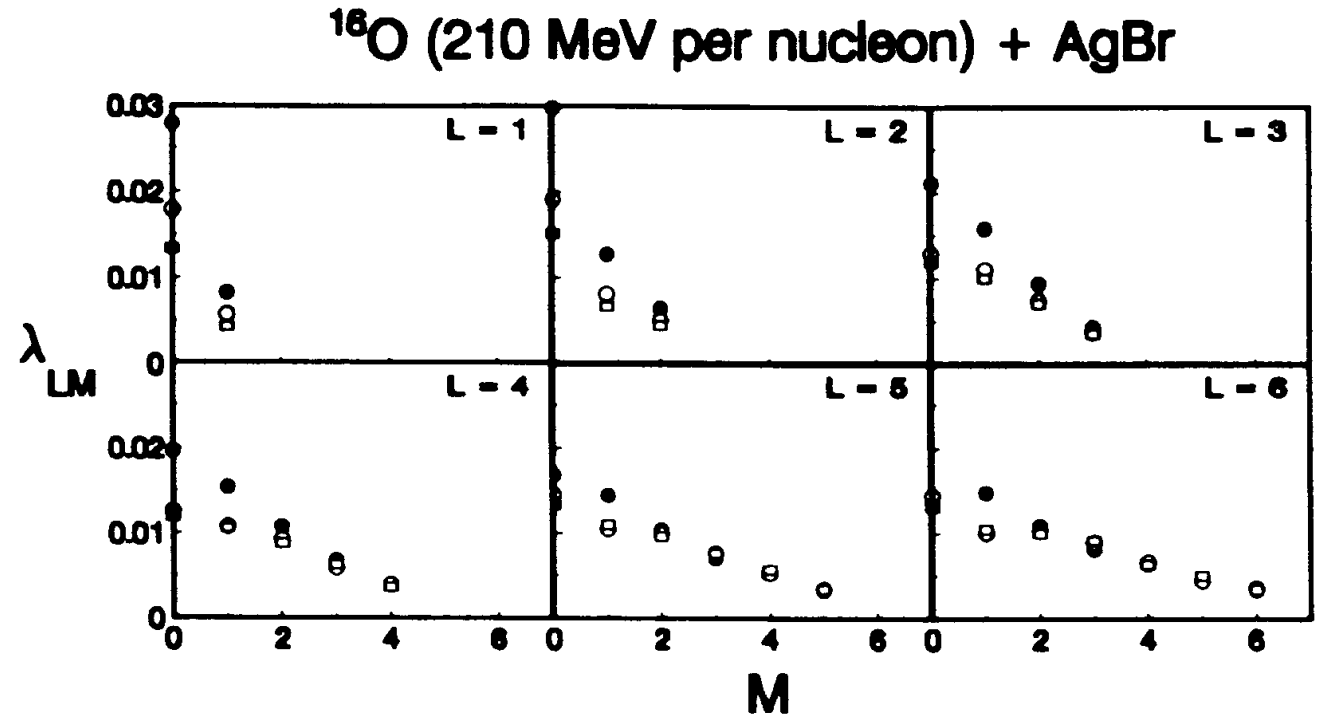

Fig. 16 


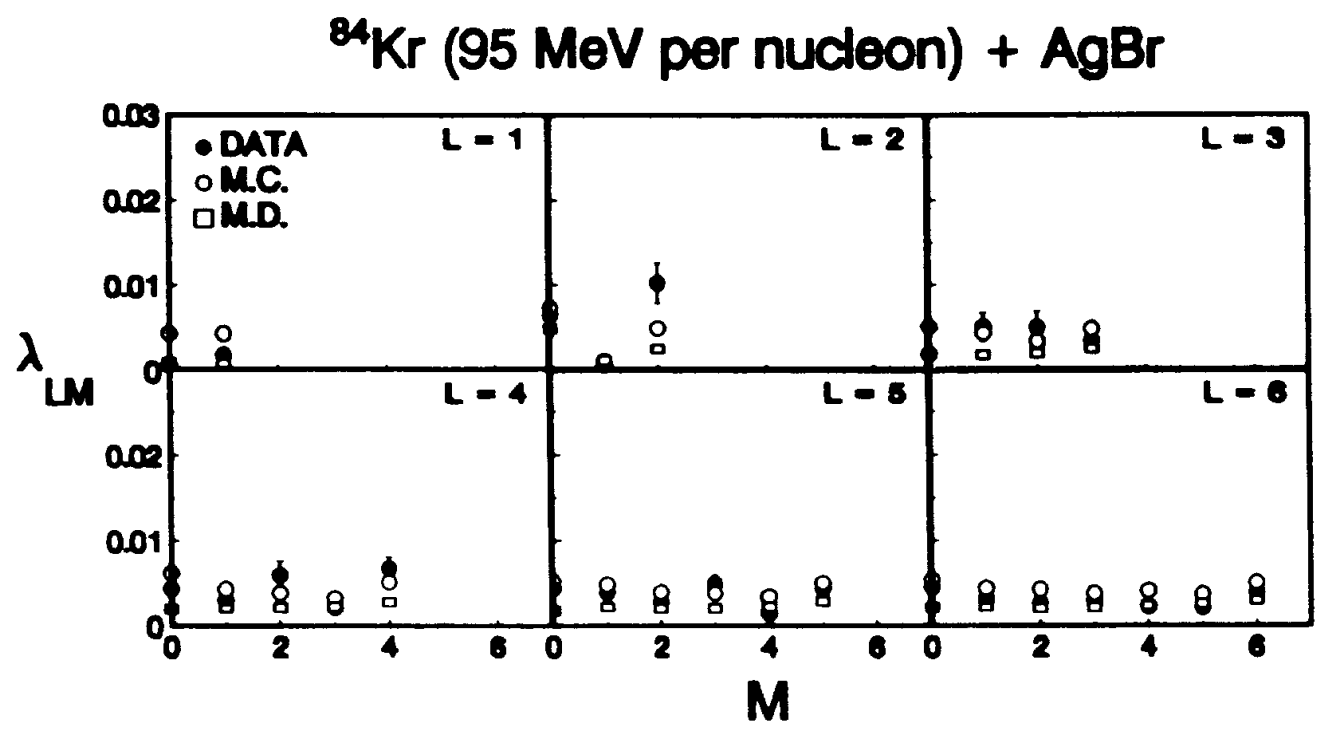

Fig. 2 


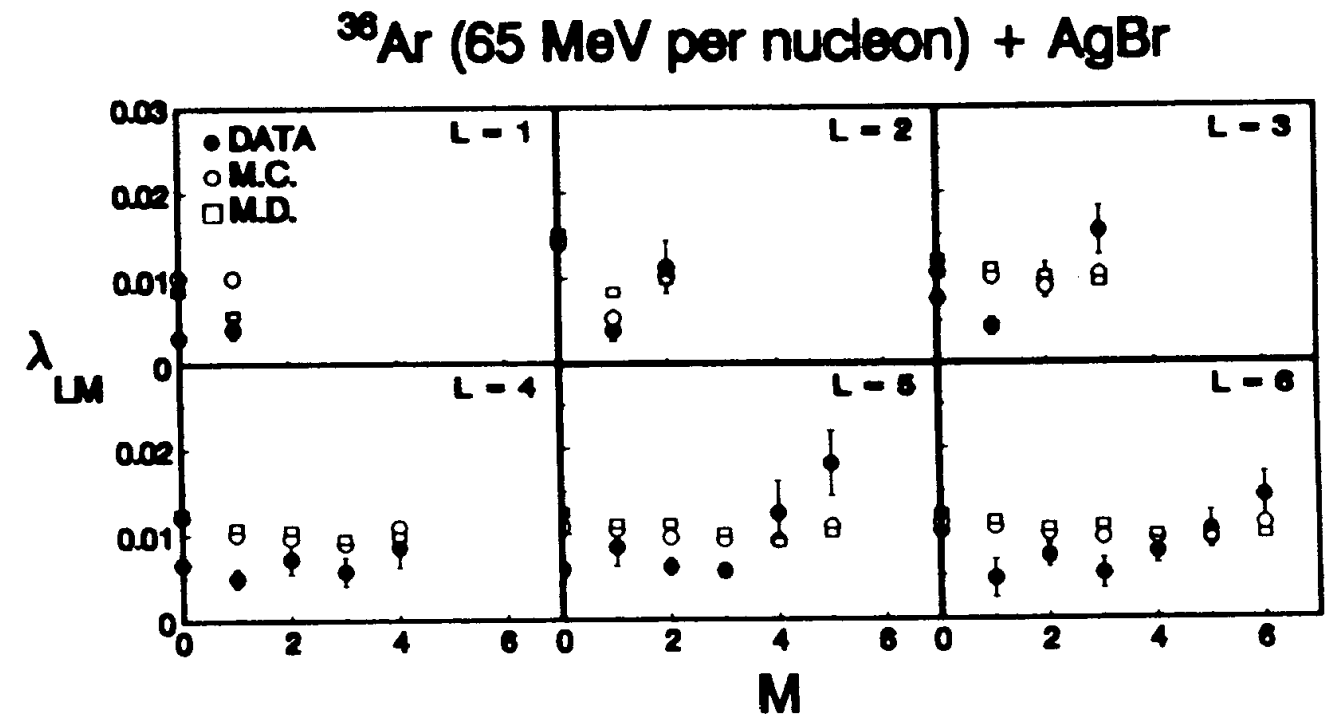

Fig. 3 


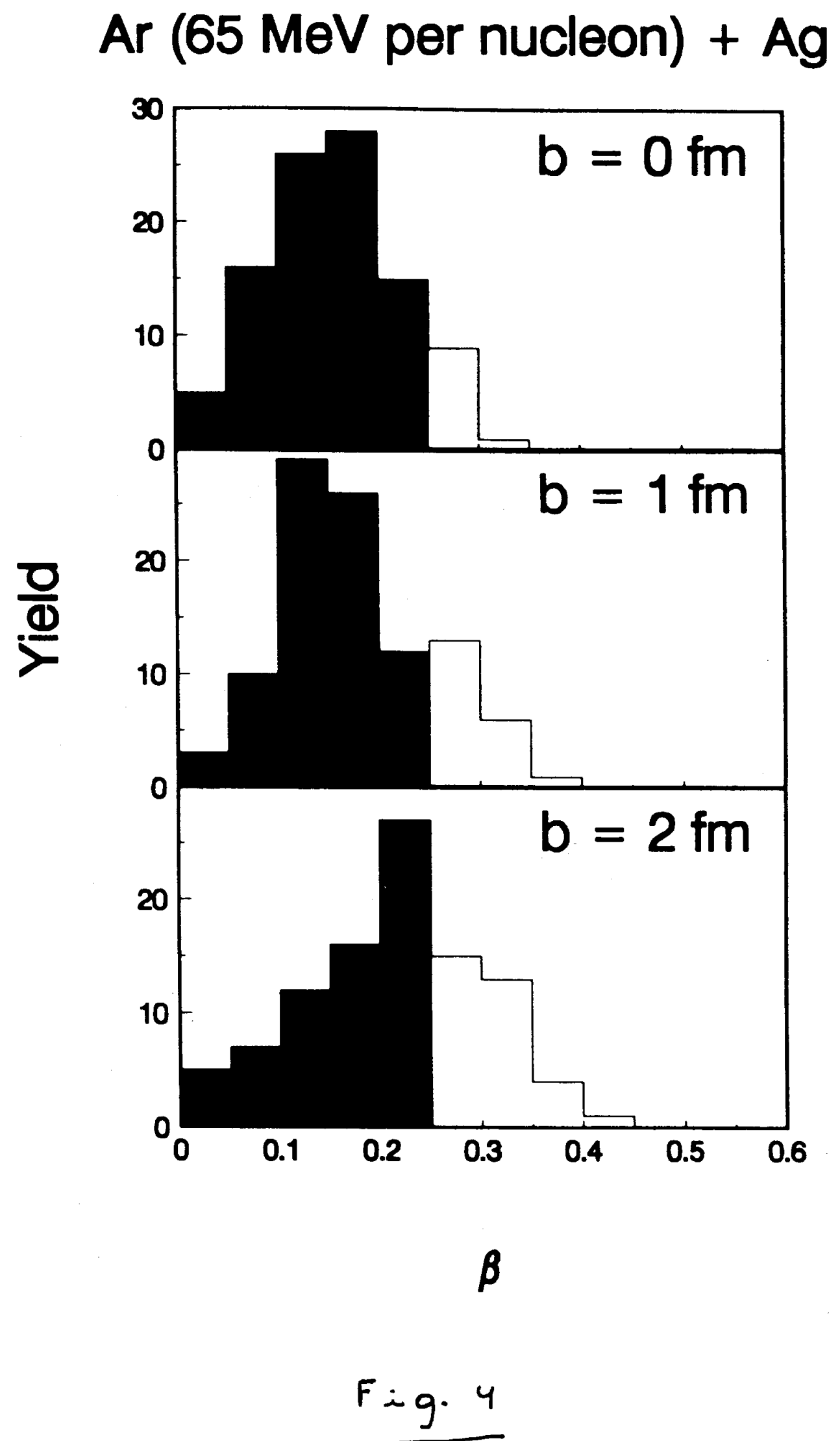




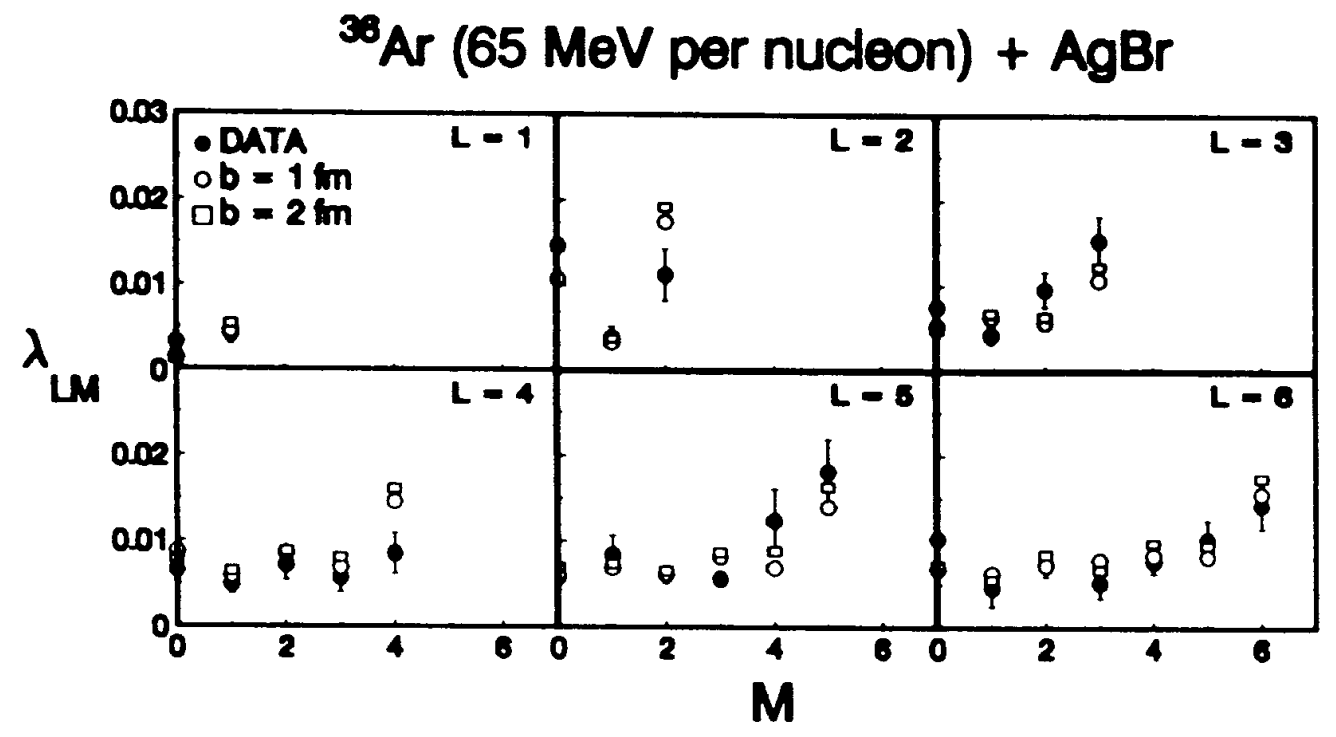

Fig. 5 
${ }^{16} \mathrm{O}$ (210 MeV per nucleon) $+\mathrm{AgBr}$

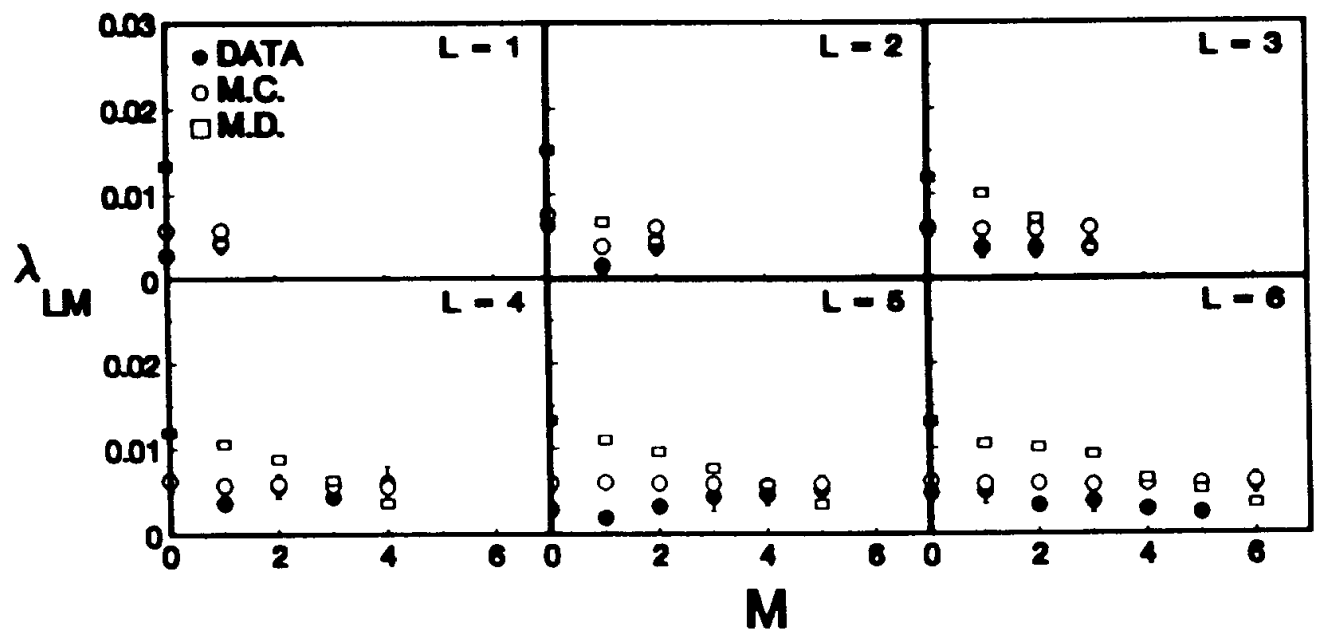

Fig. 6 


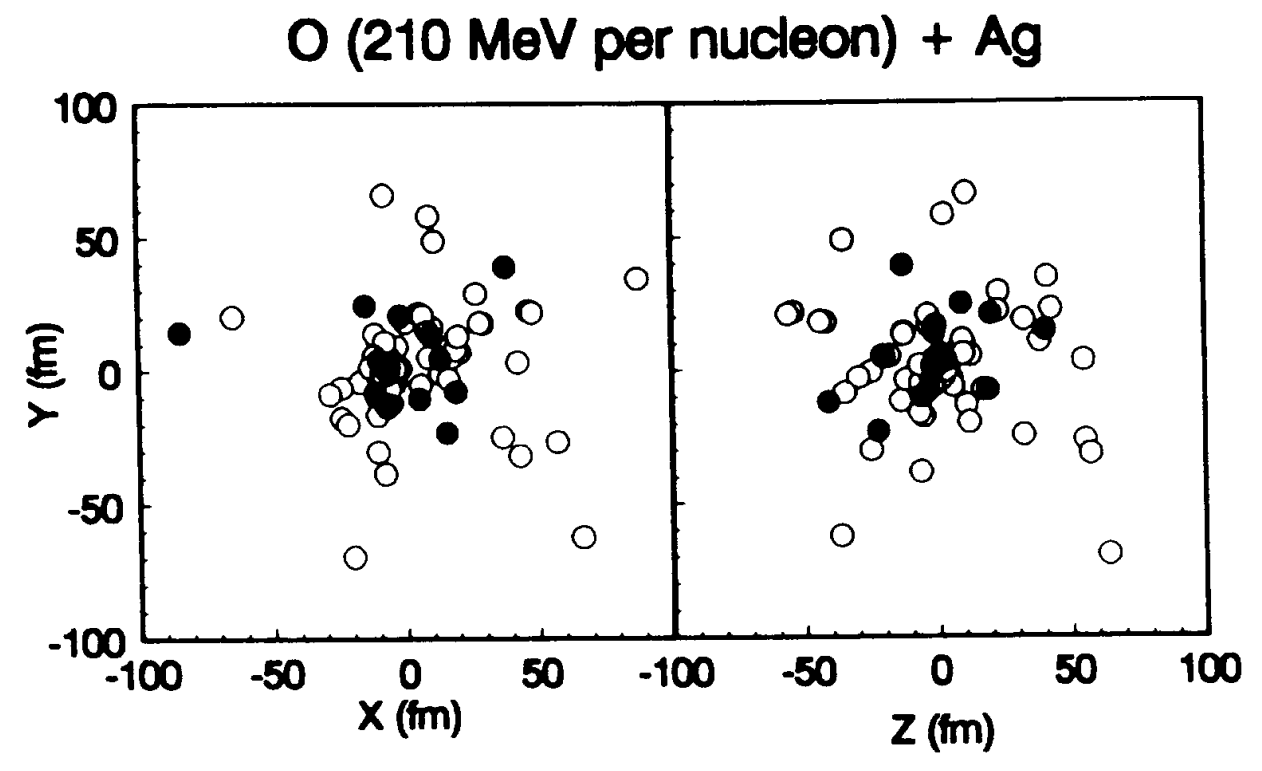

Fig. 7 


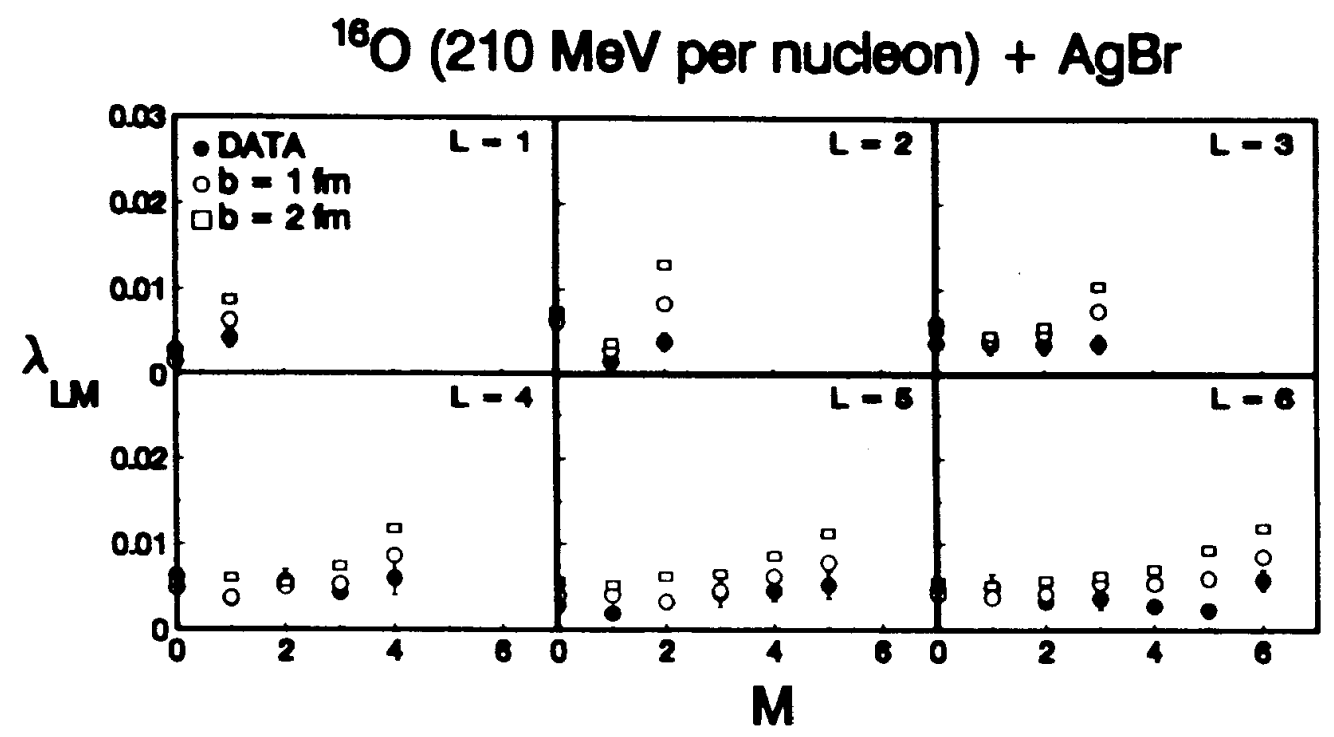

Fig. 8 
